\title{
Reduced Heart Rate Response during Drug-Induced Stress Is Related to the Severity of Perfusion Defect
}

\author{
Amr Kamal*, Eswaihal Hassan, Sahar Azab, Ahmed Abdelaaty \\ Cardiology Department, Faculty of Medicine, University of Alexandria, Alexandria, Egypt \\ Email: ^AMR.KAMAL@alexmed.edu.eg
}

How to cite this paper: Kamal, A., Hassan, E., Azab, S. and Abdelaaty, A. (2021) Reduced Heart Rate Response during DrugInduced Stress Is Related to the Severity of Perfusion Defect. World Journal of Cardiovascular Diseases, 11, 539-551.

https://doi.org/10.4236/wjcd.2021.1111051

Received: October 7, 2021

Accepted: November 27, 2021

Published: November 30, 2021

Copyright (c) 2021 by author(s) and Scientific Research Publishing Inc. This work is licensed under the Creative Commons Attribution International License (CC BY 4.0).

http://creativecommons.org/licenses/by/4.0/

\begin{abstract}
Introduction: During the pharmacological stress test with dipyridamole, a normal hemodynamic response is slightly reduced blood pressure and raised heart rate (HR). However, sometimes the HR response is reduced. This study investigated the relationship between the HR response during the dipyridamole stress test and the severity of the perfusion defects using Thallium 201 myocardial perfusion imaging. Methods: We enrolled 50 patients undergoing dipyridamole stress at the nuclear cardiology Lab, Main University Hospital of Alexandria. Standard dipyridamole protocol (infusion over $4 \mathrm{~min}$ ) and standard thallium 201 protocol ( 2 min after dipyridamole infusion) were followed. If the heart rate (HR) ratio (peak HR/rest HR) was 1.20 or less, it was considered a reduced response. Total perfusion defect (TPD), summed stress score (SSS), summed rest score (SRS), and summed difference score (SDS) for myocardial perfusion were calculated. Patients with reduced HR response and normal HR responses were compared and independent predictors of reduced HR response were determined. Results: About $56 \%$ of patients had reduced HR response; which included a high number of patients with a history of dyslipidemia and diabetes mellitus. The reduced HR response group showed lower SSS, SRS. The analysis showed that the independent predictors of reduced HR response were rest HR, SSS, and diabetes mellitus. Conclusions: Reduction in the HR response during the dipyridamole-induced stress test is related to the severity of perfusion defect, diabetes mellitus, and Dyslipidemia.
\end{abstract}

\section{Keywords}

Myocardial Perfusion Scintigraphy, Dipyridamole, Heart Rate 


\section{Introduction}

A cardiac stress test is a diagnostic test conducted in a controlled clinical environment to measure the heart's ability to respond to external stress induced either by exercise or by pharmacological stimulation. In the test, coronary circulation at rest and the maximum cardiac exertion are compared to unveil the abnormal blood flow to the myocardium.

In patients unable to perform the required physical exercise for the test due to physical limitations or other conditions, stress is induced by pharmacologic stimulation. 1 Vasodilators like dipyridamole, adenosine, or regadenoson are used for pharmacologic-stress tests to imitate a similar cardiac response to exercise. These vasodilators increase the myocardial perfusion to reveal ischemic regions. Myocardial perfusion and myocardial function are assessed using an imaging modality with a contrast medium (myocardial perfusion imaging; MPI). Maximum stress, whether induced by physical activity or drug-induced, leads to maximum coronary blood flow. The resultant coronary blood flow reveals inconsistency in blood flow between normal and stenosed arteries. The increased oxygen demand in response to exercise, or vasodilator effects of a pharmacologic stress agent creates the required stress to attain the coronary flow.

The resting coronary flow can remain normal in coronary artery disease (CAD) despite high-grade stenosis due to reduced distal arterial resistance in the stenosed arteries to allow normal perfusion. Putting heart in stressed conditions can help to detect CAD. When the pharmacologic stressor is administered to patients, myocardial regions supplied by normal or near-normal arteries have increased blood flow, while the stenosed arteries have lower blood flow.

Dipyridamole is a thromboxane synthase inhibitor and a lipophilic pyrimidine. It leads to indirect vasodilatation due to increased myocardial oxygen demand when administered intravenously. Dipyridamole inhibits cellular uptake of endogenous adenosine and also inhibits adenosine breakdown. This increases local adenosine concentration leading to indirect vasodilatation [1]. In patients with coronary artery diseases, regional heterogeneity is generated in coronary artery flow. The coronary arterial blood flow increases approximately thrice the resting values in normal subjects. Whereas, in the tissue supplied by a stenosed coronary artery the hyperaemic flow is reduced. The difference in the blood flow causes heterogeneity of tracer uptake on perfusion images, ultimately detecting the abnormalities.

Dipyridamole is associated with a lower frequency of side effects and is cost-effective compared to adenosine and regadenoson. The heart rate (HR) is slightly raised and systolic and diastolic blood pressures are reduced during the dipyridamole-induced stress test [2] [3]. Although some studies have associated the reduced HR response observed in dipyridamole induced stress test to increased risk of cardiac death [4] [5] [6] [7]. There have been no studies to understand the abnormal hemodynamic response and its relation to the perfusion defect. 
This study investigated HR response to dipyridamole and its association with the severity of perfusion defect, along with demographic variables, hemodynamic factors, and non-gated SPECT variables in patients undergoing myocardial perfusion scintigraphy. This study investigated the relationship between the reduced HR response during the dipyridamole-induced stress test and the severity of the perfusion defect using the Thallium 201 SPECT study.

\section{Methods}

The study was conducted at the cardiology department of the main university hospital of Alexandria. The protocol was approved by the main university hospital, University of Alexandria, Egypt prior to study commencement.

\subsection{Study Population}

A total of 50 consecutive patients undergoing SPECT with pharmacological stress (dipyridamole) at the nuclear cardiology Lab of the cardiology department of the main university hospital of Alexandria were enrolled in the study. Interviews were conducted at the time of SPECT to collect clinical information that included main complaints of patients, smoking habits, dyslipidemia, history of hypertension (history of BP $>140 / 90 \mathrm{mmHg}$ with or without the use of the antihypertensive drug), history of diabetes mellitus (insulin, oral hypoglycemic medications), previous myocardial infarction, family history. Informed consent was obtained from all the patients before enrollment in the study.

Patients with severe valvular heart disease or congestive heart failure (NHYA class III/IV), history of CABG, and bronchial asthma, and pregnant women were excluded from the study.

\subsection{Dipyridamole Protocol}

Dipyridamole was administered at $0.56 \mathrm{mg} / \mathrm{kg}$ intravenously over a period of 4-min with continuous monitoring using 12-lead ECG. Thallium-201 was injected $2 \mathrm{~min}$ after the dipyridamole infusion. During 6 min of administration of dipyridamole and thallium-201, at rest HR and blood pressure, were monitored every min. If patients had severe chest pain or bronchospasm during the test, sublingual nitrate was administered; if it remained unresponsive Aminophylline (120 - $240 \mathrm{mg}$ ) was administered.

HR response is calculated as below:

$$
\mathrm{HR} \text { response }=\mathrm{HR} \text { at peak } / \mathrm{HR} \text { at rest }
$$

(Peak HR = highest HR during monitoring)

An HR response lower than or equal to 1.20 was considered reduced HR response. 5 Patients were instructed to abstain from smoking, caffeinated food, and beverages, or methyl-xanthene products for $12 \mathrm{~h}$ before the test. Also, doses of beta-blockers, calcium channel blockers, and nitrates were withheld for at least 24 - $48 \mathrm{~h}$ before the study. 


\subsection{SPECT Protocol}

SPECT was performed using contrast-thallium 201 (Tl-201). Patients were injected with (3 - $3.5 \mathrm{ml}$ Curi) of Tl-201 after 2 minutes of finishing dipyridamole infusion. Stress acquisition was started within 20 minutes after Tl-201 injection. The resting image acquisition was started $3-4 \mathrm{~h}$ after the stress acquisition with reinjection of ( $1-1.5 \mathrm{ml}$ Curi) of thallium 201. Imaging was started within 20 minutes of injecting Tl-201. The American Society of Nuclear Cardiology guidelines were followed for image acquisition and processing. Standard filtered back projection (Butterworth filter with a critical frequency of 4, order 5) was used to reconstruct all the raw data acquired.

\subsection{Imaging and Image Analysis}

Images were analyzed using a previously validated automated program. The extent and severity of LV perfusion defect size and the extent of reversible (ischemia) or fixed (scar) resting hypoperfusion was determined. The resultant transaxial slices were reoriented perpendicular to the heart's long axis to yield long axis and short axis tomograms. No attenuation or scatter correction was used. Non-gated SPECT images were used for the assessment of semi quantitative myocardial perfusion in 20 segments. The assessment was performed according to a five-point scoring system (0-normal; 1-slight reduction of uptake; 2 -moderate reduction; 3-severe reduction; 4-absent uptake). The summed scores-summed stress score (SSS), summed rest scores (SRS), and summed difference score (SDS, the difference between SSS and SRS) were derived automatically. Image analysis was performed by a panel of two experts.

\subsection{Statistical Analysis}

The data were analyzed by multivariable logistic regression analysis. Data were analyzed using IBM SPSS software package version 20.0. Qualitative data were described using the number and percent. Quantitative data were described using a range (minimum and maximum) mean, standard deviation, and median. A comparison between normal HRR and reduced HRR was tested using the Chi-square test. The distributions of quantitative variables were tested for normality using the Kolmogorov-Smirnov test, Shapiro-Wilk test and D'Agstino test, Histogram, and QQ plot. If data were normally distributed, parametric tests were applied. If the data were abnormally distributed, non-parametric tests were used. For normally distributed data, a comparison between normal HRR and reduced HRR was done using an independent t-test while abnormally distributed data were assessed using the Mann-Whitney Test. The significance level was at $5 \%$.

\section{Results and Discussion}

Demographic data and the HR responses of the patients are presented in Table 1. Prevalence of hypertension, myocardial infarction history, dyslipidemia, and 
Table 1. Demographic data of the study population and HR responses.

\begin{tabular}{|c|c|c|c|c|c|}
\hline & $\begin{array}{c}\text { Total } \\
\text { population } \\
(\mathrm{n}=50)\end{array}$ & $\begin{array}{c}\text { Normal } \\
\text { HRR } \\
(n=22)\end{array}$ & $\begin{array}{c}\text { Reduced } \\
\text { HRR } \\
(n=28)\end{array}$ & Test of sig. & p-value \\
\hline \multicolumn{6}{|l|}{ Age (years): } \\
\hline Range & $(38-74)$ & $(38-65)$ & $(42-74)$ & \multirow{3}{*}{$\mathrm{t}=2.718^{\star}$} & \multirow{3}{*}{$0.009^{*}$} \\
\hline Mean & 59.0 & 54.77 & 62.32 & & \\
\hline S.D. & 10.36 & 11.54 & 8.09 & & \\
\hline \multicolumn{6}{|l|}{ Gender } \\
\hline Male & $33(66.0 \%)$ & $12(54.5 \%)$ & $21(75.0 \%)$ & \multirow[t]{2}{*}{$\chi^{2}=2.297$} & \multirow[t]{2}{*}{0.130} \\
\hline Female & $17(34.0 \%)$ & $10(45.5 \%)$ & $7(25.0 \%)$ & & \\
\hline \multicolumn{6}{|l|}{ Risk factors } \\
\hline Diabetes mellitus & $24(48.0 \%)$ & $8(36.4 \%)$ & $16(57 \%)$ & $x^{2}=5.541$ & $0.019^{*}$ \\
\hline Hypertension & $33(66.0 \%)$ & $15(68.2 \%)$ & $18(64.3 \%)$ & $\chi^{2}=0.083$ & 0.773 \\
\hline Dyslipidemia & $26(52.0 \%)$ & $10(45.5)$ & $16(57.1 \%)$ & $\chi^{2}=0.674$ & 0.412 \\
\hline Smoking & $21(42.0 \%)$ & $7(31.8 \%)$ & $14(50.0 \%)$ & $\chi^{2}=1.672$ & 0.196 \\
\hline $\mathrm{F} / \mathrm{H}$ of early CAD & $12(24.0 \%)$ & $5(22.7 \%)$ & $7(25.0 \%)$ & $\chi^{2}=0.035$ & 0.852 \\
\hline Previous MI & $28(56.0 \%)$ & $11(50.0 \%)$ & $17(60.7 \%)$ & $\chi^{2}=0.574$ & 0.449 \\
\hline \multicolumn{6}{|l|}{ Symptoms } \\
\hline No chest pain & $11(22.0 \%)$ & $4(18.2 \%)$ & $7(25.0 \%)$ & & \\
\hline Atypical chest pain & $22(44.0 \%)$ & $10(45.5 \%)$ & $12(42.9 \%)$ & \multirow[t]{2}{*}{$x^{2}=0.344$} & \multirow[t]{2}{*}{0.842} \\
\hline Typical chest pain & $17(34.0 \%)$ & $8(36.4 \%)$ & $9(32.1 \%)$ & & \\
\hline $\begin{array}{l}\text { Significant } \\
\text { ECG changes }\end{array}$ & $15(30.0 \%)$ & $4(18.2 \%)$ & $11(39.3 \%)$ & $x^{2}=2.613$ & 0.106 \\
\hline
\end{tabular}

${ }^{*}$ Statistically significant.

diabetes mellitus was $66 \%, 56 \%, 52 \%$, and $48 \%$, respectively in the total patients (Figure 1). $\mathrm{P} \leq 0.001$ was considered statistically significant.

Of the 50 patients, normal HR response was observed in 22 patients (44\%) and reduced HR response was observed in 28 patients (56\%). There was no difference observed in the reduced HR response group and normal HR response group based on gender, family history, smoking, history of myocardial infarction, and Dyslipidemia. Also, there was no difference in the symptoms observed between reduced HR response group and normal HR response group as the reduced HR response was asymptomatic. In the reduced HR response group, a clinically significant number of patients with diabetes mellitus compared to the normal HR response group [16 patients (57\%) vs. 8 patients (36.4\%); $\mathrm{p}=0.019$ ].

\subsection{Clinical Symptoms}

Eleven of the studied patients (22\%) presented without chest pain, seven of them 
(25\%) were with reduced heart rate response in comparison to around (18\%) with normal heart rate response, 22 of the studied patients (44\%) presented with atypical chest pain, 17 patients (34\%) presented with typical chest pain. There was no statistically significant difference was found by means of symptoms between the normal heart rate response and the reduced heart rate response groups, however. The reduced HR response group tends to be more asymptomatic (Table 1) (Figure 2).

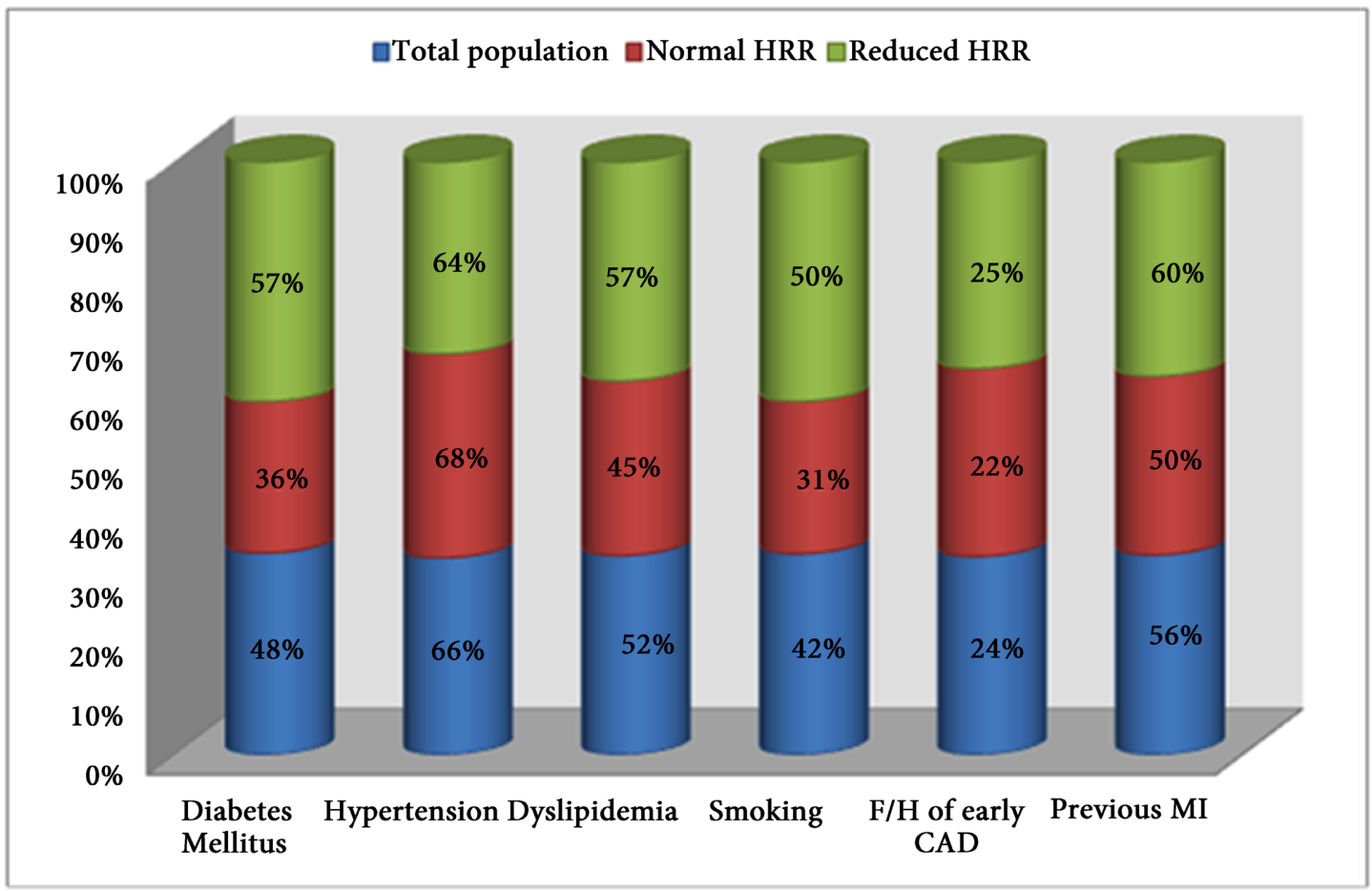

Figure 1. Distribution of patients according to risk factors.

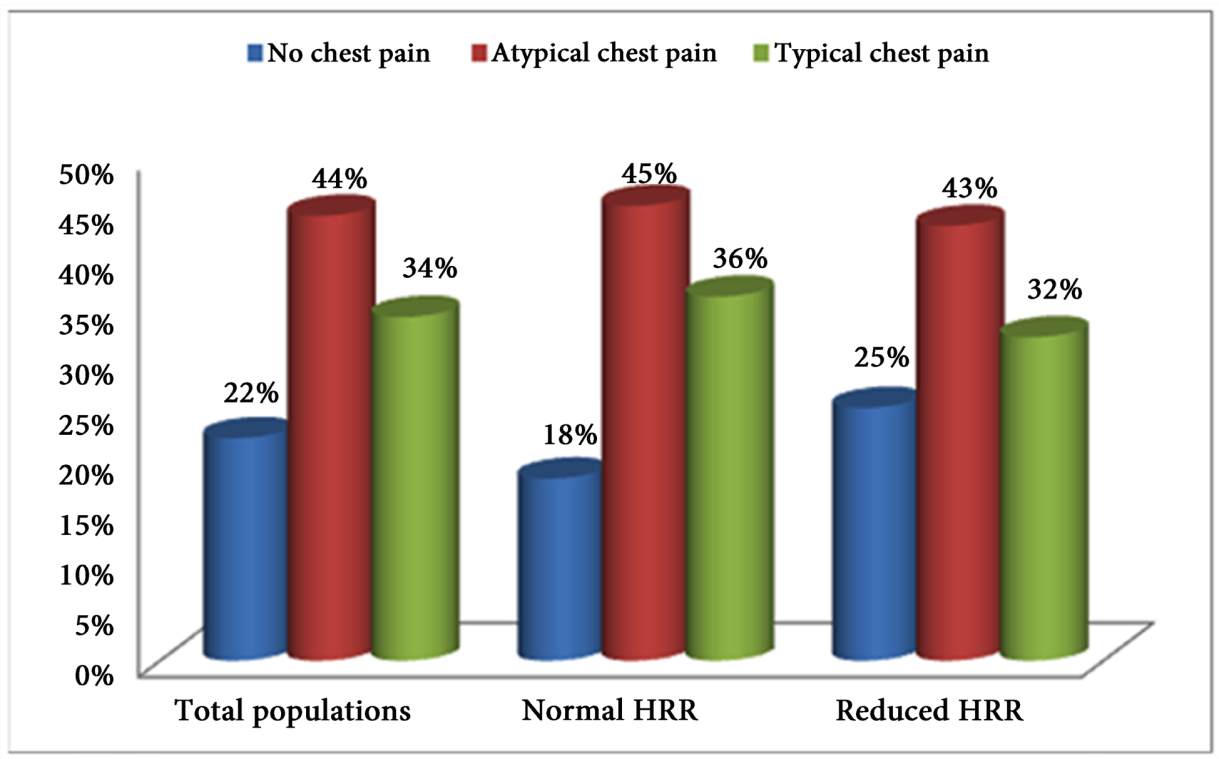

Figure 2. Distribution of patients as per the presented clinical symptoms. 


\subsection{ECG Changes}

About 15 patients (30\%) had significant ST-segment depression (11 patients had reduced HR response) and no patient had ST-segment elevation (Table 1). However, there was no statistical significance difference in ECG changes between the normal and reduced HR response groups.

\subsection{Hemodynamic Parameters}

The hemodynamic responses observed in the reduced HR response group and the normal HR response groups are presented in Table 2. As per the experts' semi-quantitative assessment, SSS, SRS, TPD, and severity of perfusion defect were significantly higher in the reduced HR response group compared to the normal HR response group, whereas there was no difference in SDS. SPECT parameters in the reduced HR response group and normal HR response group are presented in Table 3 . Patients with reduced HR had significantly higher reversible, fixed perfusion defects or both (Table 3). The SSS and SRS in the reduced HR were 17.5 and 12.1, respectively compared to 6.5 and 3.1 of the normal HR response group. Also, the mean of total perfusion defect in reduced HR response (21.39\%) was significantly higher compared to normal HR response (7.82\%) (Figure 3).

\subsection{The Severity of the Perfusion Defect}

Semi-quantitative visual interpretation of SPECT images was performed with short axis and vertical long axis tomography divided into 17 segments (Figure 4 ). The consensus of two expert observers scored each segment using a 5-point scale $(58,59)$ and categorized it into normal, mild, moderate \& severe perfusion defects (Table 3). Patients with reduced HR response showed a significantly more

Table 2. Hemodynamic response in normal HRR and RHRR groups.

\begin{tabular}{lcccc}
\hline & $\begin{array}{c}\text { Normal } \\
\text { HRR }(\mathbf{n}=22)\end{array}$ & $\begin{array}{c}\text { Reduced } \\
\text { HRR }(\mathbf{n}=28)\end{array}$ & Test of sig. & p-value \\
\hline $\begin{array}{l}\text { Hemodynamic } \\
\text { Heart rate }\end{array}$ & & & & \\
a) Rest & $75.05 \pm 14.69$ & $79.43 \pm 10.84$ & $\mathrm{t}=1.214$ & 0.231 \\
b) Stress & $94.18 \pm 16.52$ & $85.39 \pm 11.78$ & $\mathrm{t}=1.214$ & $0.033^{*}$ \\
c) Delta & $13.86 \pm 12.56$ & $12.0 \pm 7.57$ & $\mathrm{t}=0.650$ & 0.519 \\
Blood pressure & & & & \\
a) Rest systolic & $144.55 \pm 27.38$ & $138.57 \pm 25.49$ & $\mathrm{t}=0.796$ & 0.430 \\
b)Peak systolic & $137.27 \pm 24.53$ & $133.93 \pm 24.55$ & $\mathrm{t}=0.478$ & 0.635 \\
$\begin{array}{l}\text { a) Rest diastolic } \\
\text { b)Peak diastolic }\end{array}$ & $82.95 \pm 14.85$ & $83.57 \pm 11.93$ & $\mathrm{t}=0.163$ & 0.871 \\
c)Delta & $80.45 \pm 15.58$ & $84.64 \pm 15.75$ & $\mathrm{t}=0.938$ & 0.353 \\
\hline
\end{tabular}

*Statistically significant. 
Table 3. SPECT parameters in normal HRR and RHRR groups.

\begin{tabular}{lcccc}
\hline & $\begin{array}{c}\text { Normal } \\
\text { HRR (n=22) }\end{array}$ & $\begin{array}{c}\text { Reduced } \\
\text { HRR }(\mathbf{n}=28)\end{array}$ & Test of sig. & p-value \\
\hline Scoring scale & & & & \\
a) SSS & $6.50 \pm 5.98$ & $17.50 \pm 10.80$ & $\mathrm{Z}=106.50^{*}$ & $<0.001^{\star}$ \\
b) SRS & $3.14 \pm 4.0$ & $12.18 \pm 10.39$ & $\mathrm{Z}=115.0^{*}$ & $<0.001^{*}$ \\
c) SDS & $3.23 \pm 4.24$ & $4.96 \pm 4.66$ & $\mathrm{Z}=218.0^{*}$ & 0.075 \\
d) TPD & $7.82 \pm 6.34$ & $21.39 \pm 14.18$ & $\mathrm{Z}=112.50^{*}$ & $<0.001^{*}$ \\
Distribution & & & & \\
a) LAD & $6(27.3 \%)$ & $17(60.7 \%)$ & $\chi^{2}=5.547^{*}$ & $0.019^{*}$ \\
b) RCA & $10(45.5 \%)$ & $14(50.0 \%)$ & $\chi^{2}=0.102$ & 0.749 \\
c) LCX & $4(18.2 \%)$ & $13(46.4 \%)$ & $\chi^{2}=4.381^{*}$ & $0.036^{*}$ \\
d) 2 vessels & $5(22.7 \%)$ & $11(39.3 \%)$ & $\chi^{2}=1.552$ & 0.213 \\
e) 3 vessels & $0(0.0 \%)$ & $2(7.1 \%)$ & $\chi^{2}=1.637$ & FEp $=0.497$ \\
Severity & & & & \\
a) Normal & $7(31.8 \%)$ & $0(0.0 \%)$ & & $<0.001^{*}$ \\
b) Mild & $8(36.4 \%)$ & $8(28.6 \%)$ & $Z=118.50^{*}$ & \\
c) Moderate & $5(22.7 \%)$ & $3(10.7 \%)$ & & \\
d) Sever & $2(9.1 \%)$ & $17(60.7 \%)$ & & \\
\hline
\end{tabular}

*Statistically significant.

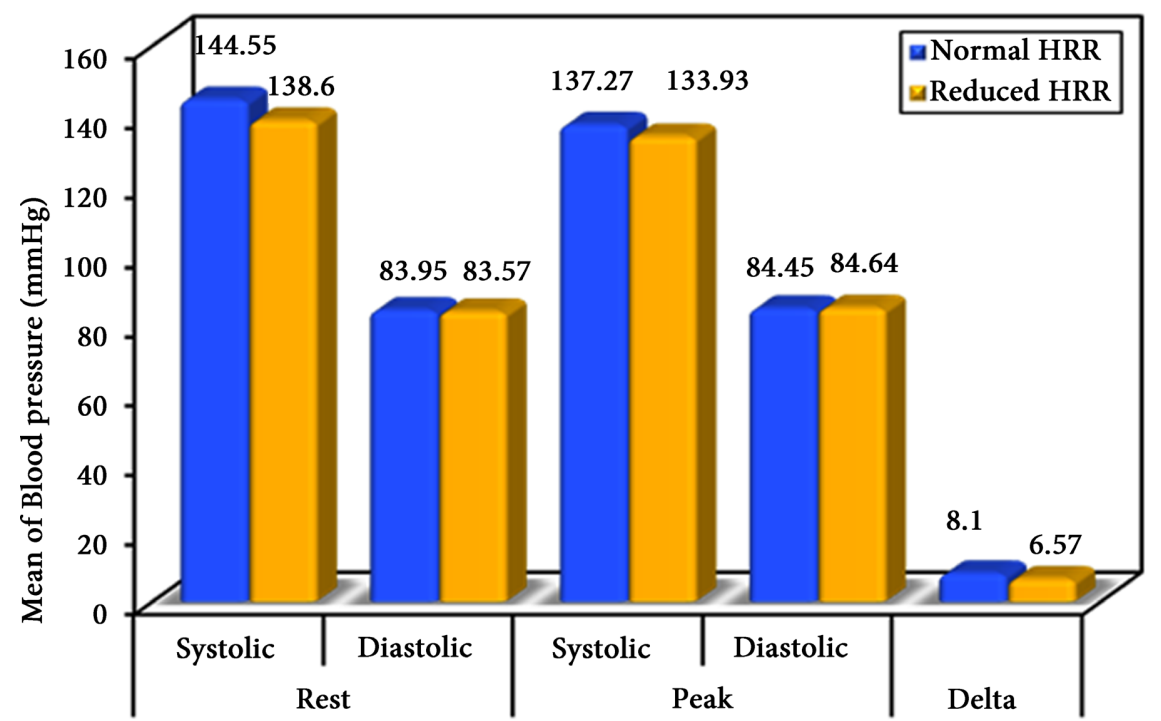

Figure 3. Blood pressure response in normal HR and reduced HR response groups.

tendency of the perfusion defect than patients with normal HR response.

Dipyridamole is an indirect vasodilator that prevents intracellular reuptake and deamination of adenosine. It causes an increase in adenosine levels [1]. 


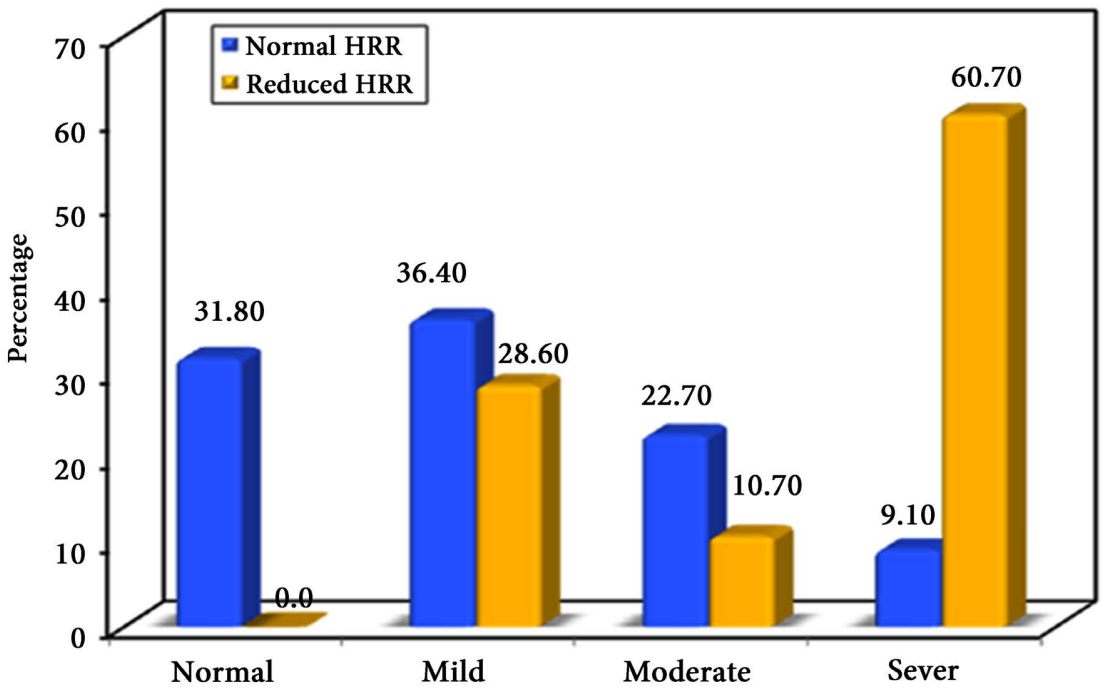

Figure 4. SPECT parameters in normal HR and reduced HR group discussion.

Dipyridamole decreases coronary artery resistance through the A2a receptor. The difference in coronary blood flow between normal and stenosed arteries is captured in the myocardial perfusion imaging [2]. A1, A2b, and A3 receptor activation consequently lead to reduced systolic and diastolic BP and elevated HR. Dipyridamole produced peripheral vasodilatation is a result of A2b receptor activation causing the hemodynamic response [2] [3]. Recently, selective A2a agonists-adenosine and regadenoson, have been shown to directly stimulate the sympathetic nervous system [1] [8] [9] [10].

In this study, the observed parameters of the severity of perfusion defect like the average SSS,SRS, and stress TPD in the patients with reduced HR response were significantly higher $(17.5,12.1,21.39$ respectively) compared to patients with normal HR response $(6.5,3.1,7.82$ respectively). This shows that HR response to dipyridamole is directly associated with the severity of perfusion defect. The panel of two expert observers compared and interpreted the SPECT images obtained in patients with reduced HR response with patients with normal HR response confirming the observation.

Although a few studies have investigated the relationship between the severity of perfusion defect and the reduced HR response, there is no dependable explanation for the relationship yet. A plausible explanation could be that the association might be directly or indirectly related to the severe involvement of the sympathetic plexus of the heart because the SDS (ischemia) in the reduced HR response group and normal HR response group showed no difference in the analysis. The severity of the perfusion defect might reflect ventricular dysfunction.

The reduced HR response to vasodilators may be due to autonomic imbalance. 8 - 10 Also, changes in regulatory G protein levels in patients with congestive heart failure may affect HR response [2] [11].

In this study, we also studied the distribution of first-order branch vessels of 
the coronary arteries. We found that the involvement of LAD and LCX in patients with reduced heart rate response was significantly higher $(60.7 \%, 46.4 \%$, respectively) than those in patients with normal heart rate response $(27.3 \%, 18.2 \%$, respectively). However, no statistically significant difference was observed in the RCA, two vessels, and three vessels coronary artery involvement between the two groups.

Although our results are significant, the sensitivity, specificity, and accuracy of SPECT MPI in our study for the detection of CAD in the distribution of first-order branch vessels were intermediate ( $71 \%, 67 \%$ and $68 \%$ respectively). Therefore, there is a need to correlate the obtained results with more accurate coronary artery distribution imaging modality like CMR and angiography. Though the sensitivity, specificity, and accuracy of the imaging modality in intermediate in this study, the observations indicate apparent contradictions requiring further investigation in a larger study.

In this study reduced HR response group had high rest HR, which is an independent predictor of reduced HR response, and is also indicator of cardiac autonomic neuropathy, reflecting preexisting dominance of sympathetic effect on sinus node [12] [13]. This might be a plausible explanation for vasodilators' minimal effect on heart rate. In addition, the highest HR in reduced HR response group can be associated with the diseases (For example diabetes mellitus) causing cardiac autonomic dysfunction.

We also observed that the patients with normal heart rate response comparatively had a slightly higher baseline and lowest systolic BP and diastolic BP. Furthermore, the mean blood pressure difference in patients with normal HR responses was higher compared to patients with reduced HR response although not statistically significant.

Some researchers have investigated the implications of an abnormal BP response to vasodilators [14]. Hatutale et al. assessed changes in the HR and BP during the dipyridamole stress and abnormal scan findings using gated technetium-99m methoxy isobutylisonitrile MPI [15]. They demonstrated that the abnormal hemodynamic response-decreased HR response and abnormal systolic BP response were significantly associated with low Left Ventricular Ejection Factor $(\mathrm{P}=0.012)$, abnormal myocardial perfusion imaging scan findings, and SSS [15]. Recent studies indicate that increases in HR could be due to direct adenosine-induced stimulation of sympathetic nervous system which may be independent of the BP [10]. Whereas some studies observed that patients with decreased systolic blood pressure more frequently had myocardial ischemia compared to patients with normal or increased systolic blood pressure [14].

HR response (ratio of peak HR and baseline HR) $\leq 1.20$ was considered abnormal in this study as per the previously defined [5] [7]. The percentage of patients with reduced HR in this study (56\%) was consistent with the observed in the other studies like Mathur et al. (64\%) [7]. We recommend further studies in a larger population to determine ideal cutoffs of HR response to vasodilators like 
dipyridamole.

Dipyridamole has been associated with adverse events like flush, headache, hypotension, abdominal pain, diarrhea, dizziness, and chest pain [16] [17]. These adverse events result due to peripheral vasodilation, hypotension, and mainly due to the stimulation of adenosine receptors and signal pathways [18]. This study did not focus on the adverse events that occurred in the patients. The most common adverse events observed were flush, chest pain, and hypotension.

There were some limitations in this study including not using the cardiovascular autonomy tests and comorbidities in our patients. The autonomy test would have revealed cardiac autonomic dysfunction. Cardiovascular autonomy tests are rarely performed in regular clinical practice but it would have helped in better understanding the abnormal HR response. The percentage of co-morbidities in our patient group was higher (For example diabetes \& hypertension). Most of these patients were referred to our clinic for the stress test. Fasting plasma glucose concentrations and fasting lipid profiles were not available. Also, information about diabetes and dyslipidemia was gathered by questionnaire.

In conclusion, the reduced HR response to the dipyridamole stress test is an independent predictor of the severity of perfusion defect (reversible defects, fixed or both), SSS, SRS, and TPD. The Reduce HR response might be due to cardiac autonomic neuropathy, which needs to be further investigated in a large sample size using cardiovascular autonomy tests.

\section{Conflicts of Interest}

The authors declare no conflicts of interest regarding the publication of this paper.

\section{References}

[1] Henzlova, M.J., Duvall, W.L., Einstein, A.J., Travin, M.I. and Verberne, H.J. (2016) ASNC Imaging Guidelines for SPECT Nuclear Cardiology Procedures: Stress, Protocols, and Tracers. Journal of Nuclear Cardiology, 23, 606-639.

https://doi.org/10.1007/s12350-015-0387-x

[2] Johnston, D.L., Daley, J.R., Hodge, D.O., Hopfenspirger, M.R. and Gibbons, R.J. (1995) Hemodynamic Responses and Adverse Effects Associated with Adenosine and Dipyridamole Pharmacologic Stress Testing: a Comparison in 2,000 Patients. Mayo Clinic Proceedings, 70, 331-336. https://doi.org/10.4065/70.4.331

[3] Iskandrian, A.S., Verani, M.S. and Heo, J. (1994) Pharmacologic Stress Testing: Mechanism of Action, Hemodynamic Responses, and Results in Detection of Coronary Artery Disease. Journal of Nuclear Cardiology, 1, 94-111. https://doi.org/10.1007/BF02940016

[4] Abidov, A., Hachamovitch, R., Hayes, S.W., Ng, C.K., Cohen, I. and Friedman, J.D. (2003) Prognostic Impact of Hemodynamic Response to Adenosine in Patients Older Than Age 55 Years Undergoing Vasodilator Stress Myocardial Perfusion Study. Circulation, 107, 2894-2899. https://doi.org/10.1161/01.CIR.0000072770.27332.75

[5] Bhatheja, R., Francis, G.S., Pothier, C.E. and Lauer, M.S. (2005) Heart Rate Response 
during Dipyridamole Stress as a Predictor of Mortality in Patients with Normal Myocardial Perfusion and Normal Electrocardiograms. The American Journal of Cardiology, 95, 1159-1164. https://doi.org/10.1016/j.amjcard.2005.01.042

[6] Kim, Y.H., Lee, K.H., Chang, H.J., Lee, E.J., Chung, H.W., Choi, J.Y. and Kim, B.T. (2006) Depressed Heart Rate Response to Vasodilator Stress for Myocardial SPECT Predicts Mortality in Patients after Myocardial Infarction. The International Journal of Cardiovascular Imaging, 22, 663-670. https://doi.org/10.1007/s10554-005-9066-3

[7] Mathur, S., Shah, A.R., Ahlberg, A.W., Katten, D.M. and Heller, G.V. (2010) Blunted Heart Rate Response as a Predictor of Cardiac Death in Patients Undergoing Vasodilator Stress Technetium-99m Sestamibi Gated SPECT Myocardial Perfusion Imaging. Journal of Nuclear Cardiology, 17, 617-624. https://doi.org/10.1007/s12350-010-9242-2

[8] Hage, F.G., Perry, G., Heo, J. and Iskandrian, A.E. (2010) Blunting of the Heart Rate Response to Adenosine and Regadenoson in Relation to Hyperglycemia and the Metabolic Syndrome. The American Journal of Cardiology, 105, 839-843. https://doi.org/10.1016/j.amjcard.2009.11.042

[9] Hage, F.G., Heo, J., Franks, B., Belardinelli, L., Blackburn, B., Wang, W. and Iskandrian, A.E. (2009) Differences in Heart Rate Response to Adenosine and Regadenoson in Patients with and without Diabetes Mellitus. American Heart Journal, 157, 771-776. https://doi.org/10.1016/j.ahj.2009.01.011

[10] Dhalla, A.K., Wong, M.Y., Wang, W.Q., Biaggioni, I. and Belardinelli, L. (2006) Tachycardia Caused by $\mathrm{A}_{2 \mathrm{~A}}$ Adenosine Receptor Agonists Is Mediated by Direct Sympathoexcitation in Awake Rats. The Journal of Pharmacology and Experimental Therapeutics, 316, 695-702. https://doi.org/10.1124/jpet.105.095323

[11] Triposkiadis, F., Karayannis, G., Giamouzis, G., Skoularigis, J., Louridas, G. and Butler, J. (2009) The Sympathetic Nervous System in Heart Failure: Physiology, Pathophysiology, and Clinical Implications. Journal of the American College of Cardiology, 54, 1747-1762. https://doi.org/10.1016/j.jacc.2009.05.015

[12] Aaty, A.A., El-DeenZaky, A.T., Lotfy, M.I. and Fathy, A.M. (2015) Heart Rate Response to Dypiridamole Stress in Relation to Perfusion and Function during Gated Technecium Tc99 M Sestamibi SPECT Study. Journal of Cardiology \& Current Research, 2, Article ID: 00063. https://doi.org/10.15406/jccr.2015.02.00063

[13] Theres, H., Romberg, D., Leuthold, T., Borges, A.C., Stangl, K. and Baumann, G. (1998) Autonomic Effects of Dipyridamole Stress Testing on Frequency Distribution of RR and QT Interval Variability. Pacing and Clinical Electrophysiology, 21, 2401-2406. https://doi.org/10.1111/j.1540-8159.1998.tb01190.x

[14] Witbrodt, B., Goyal, A., Kelkar, A.A., Dorbala, S., Chow, B.J., Di Carli, M.F. and Shaw, L.J. (2017) Prognostic Significance of Blood Pressure Response during Vasodilator Stress Rb-82 Positron Emission Tomography Myocardial Perfusion Imaging. Journal of Nuclear Cardiology, 24, 1966-1975.

https://doi.org/10.1007/s12350-016-0569-1

[15] Hatutale, A., Vorster, M., Ankrah, A.O., Rheeder, P. and Sathekge, M.M. (2013) Association of Hemodynamic Response during Dipyridamole Stress Testing with 99mTc-MIBI SPET Myocardial Perfusion Image Findings. Hellenic Journal of Nuclear Medicine, 16, 181-185.

[16] Dubrey, S.W., Bomanji, J.B., Noble, M.I. and Jewkes, R.F. (1993) Safety of Intravenous Dipyridamole Thallium Myocardial Perfusion Imaging: Experience in 435 Patients. Nuclear Medicine Communications, 14, 303-309.

https://doi.org/10.1097/00006231-199304000-00171 
[17] Meyers, A.M., Topham, L., Ballow, J., Totah, D. and Wilke, R. (2002) Adverse Reactions to Dipyridamole in Patients Undergoing Stress/Rest Cardiac Perfusion Testing. Journal of Nuclear Medicine Technology, 30, 21-24.

[18] Johnson, S.G. and Peters, S. (2010) Advances in Pharmacologic Stress Agents: Focus on Regadenoson. Journal of Nuclear Medicine Technology, 38, 163-171. https://doi.org/10.2967/jnmt.109.065581 